\title{
Personalised Advertising Supported by Agents
}

\author{
Bruno Veloso $^{1,2}$, Luís Sousa ${ }^{1}$ and Benedita Malheiro ${ }^{1,2}$ \\ 1 Instituto Superior de Engenharia do Porto, Instituto Politécnico do Porto, Porto, Portugal \\ 2 INESC TEC - INESC Technology and Science (formerly INESC Porto) \\ \{bruno.miguel.veloso, letvsousa\} @gmail.com, mbm@isep.ipp.pt
}

\begin{abstract}
This paper reports the development of a B2B platform for the personalization of the publicity transmitted during the program intervals. The platform as a whole must ensure that the intervals are filled with ads compatible with the profile, context and expressed interests of the viewers.

The platform acts as an electronic marketplace for advertising agencies (content producer companies) and multimedia content providers (content distribution companies). The companies, once registered at the platform, are represented by agents who negotiate automatically the price of the interval timeslots according to the specified price range and adaptation behaviour. The candidate ads for a given viewer interval are selected through a matching mechanism between ad, viewer and the current context (program being watched) profiles. The overall architecture of the platform consists of a multiagent system organized into three layers consisting of: $(i)$ interface agents that interact with companies; (ii) enterprise agents that model the companies, and (iii) delegate agents that negotiate a specific ad or interval. The negotiation follows a variant of the Iterated Contract Net Interaction Protocol (ICNIP) and is based on the price/s offered by the advertising agencies to occupy the viewer's interval.
\end{abstract}

Keywords: Multiagent system, B2B, Multimedia, Brokerage, Profile Matching, Fixed ICNIP, Web Services.

\section{INTRODUCTION}

Media content personalisation has been addressed by several projects such as MiSPOT, NoTube, LinkedTV or HBB-NEXT. MiSPOT proposes a non-invasive and fully personalized form of advertising, using semantic reasoning techniques to select advertisements suited to the preferences, interests and needs of each viewer [1]. NoTube defines a flexible end-to-end architecture for the personalised creation, distribution and consumption of TV content [2]. LinkedTV focuses on integrating TV and Web contents based on user personalisation and contextualisation [3]. HBB-NEXT goal is to mix broadcast, Internet and user-generated content by adopting user-centric technologies to enrich the TV-viewing experience with social networking, multiple device access and group-tailored recommendations [4]. 
The work described in this paper reports on the Business-to-Business (B2B) MultiMedia Brokerage (MMB) platform for media content personalisation under development $[5,6]$. The personalisation is based on the profiles of viewers and media components (ads and intervals). The application domain is the personalization of advertising intervals, i.e., the content of viewer intervals will be negotiated to ensure an ad alignment compatible with the current context, expressed interests and previous interactions of the viewer. It is expected that, in the future, advertising agencies and media content distributors use the platform to personalize advertising and perform niche segmentation.

The platform acts as an electronic market that supports the automated trading between the advertising agencies (producers) and the content providers (distributors). The companies, once registered at the platform, are represented by agents that negotiate items automatically according to the specified negotiation behaviour, i.e., the price range and adaptation tactics. Distributors act as sellers and the producers as buyers of interval timeslots. The negotiation follows a variant of the Iterated Contract Net Interaction Protocol (ICNIP) [10] - the Fixed ICNIP - and is based on the price/s offered by producers to occupy the interval timeslots.

This paper is organised in four sections: the introductory section, the development section, covering the system architecture and functionalities, the tests and results section and the conclusions section.

\section{Multimedia Brokerage Platform}

The brokerage platform is a multiagent system organized into three layers: interface, enterprise and market layers. Fig. 1 displays an overview of the system architecture.

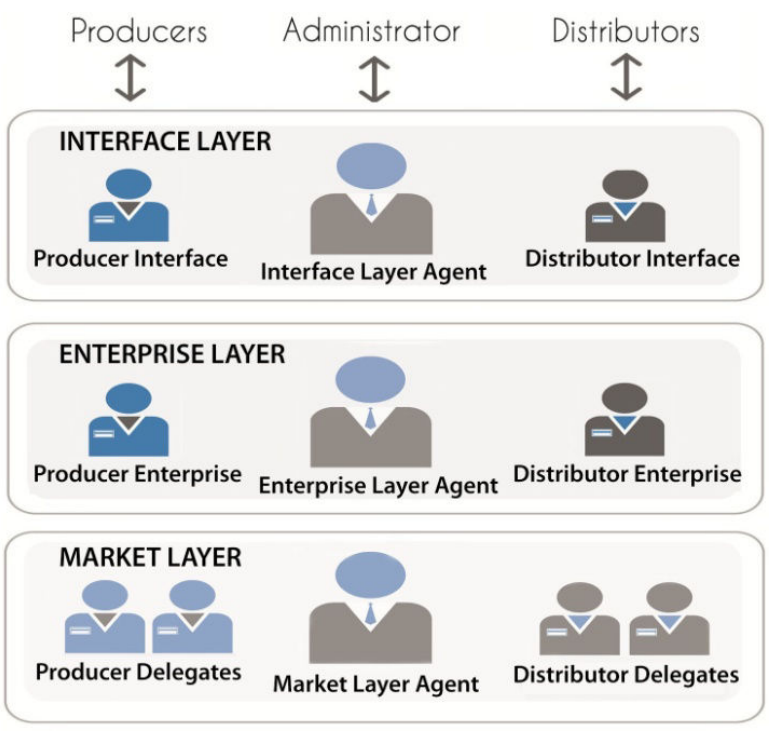

Fig. 1. - MMB platform architecture 
The agents of the platform are divided into four types: $(i)$ interface agents to interface with businesses; (ii) enterprise agents that model the businesses; (iii) market delegate agents dedicated to specific ad or interval negotiations on behalf of enterprise agents; and (iv) layer manager agents (interface, enterprise and market layer agents). Each business (producer or distributor enterprise) is represented at the platform by: $(i)$ an enterprise interface agent, which exposes a Web service with a set of interface operations, located in first layer, (ii) the enterprise agent that models the enterprise at platform, which exposes a Web service with a set of operations for the agents of the other layers, residing in the intermediate layer; and (iii) an undetermined number of delegates involved in specific negotiations. Table 1 shows the set of Web service operations exposed by the platform agents.

Table 1. - Platform agent Web services and operations

\begin{tabular}{|c|c|c|c|c|}
\hline \multirow[t]{2}{*}{ Interface Layer } & Service Type & Producer & Distributor & Layer \\
\hline & Operations & $\begin{array}{l}\text { SetAd } \\
\text { SetAdProfile } \\
\text { GetAdResults } \\
\text { RemoveAd }\end{array}$ & $\begin{array}{l}\text { SetInterval } \\
\text { SetIntervalProfile } \\
\text { SetViewerProfile } \\
\text { GetIntervalResults } \\
\text { RemoveInterval }\end{array}$ & $\begin{array}{l}\text { CreateAgent } \\
\text { KillAgent }\end{array}$ \\
\hline \multirow[t]{2}{*}{ Enterprise Layer } & Service Type & Producer & Distributor & Layer \\
\hline & Operations & $\begin{array}{l}\text { GetAdProfile } \\
\text { RemoveAd } \\
\text { SetAdProfile } \\
\text { SetAdResult } \\
\text { SetAd } \\
\text { GetAd } \\
\text { GetAdResults } \\
\text { GetProduct } \\
\end{array}$ & $\begin{array}{l}\text { GetIntervalProfile } \\
\text { SetViewerProfile } \\
\text { SetIntervalResult } \\
\text { RemoveInterval } \\
\text { SetInterval } \\
\text { GetIntervalResults } \\
\text { GetInterval } \\
\text { SetIntervalProfile }\end{array}$ & $\begin{array}{l}\text { CreateAgent } \\
\text { KillAgent }\end{array}$ \\
\hline \multirow[t]{2}{*}{ Market Layer } & Service Type & Producer & Distributor & Layer \\
\hline & Operations & & & $\begin{array}{l}\text { CreateProducerDelegate } \\
\text { CreateDistributorDelegate } \\
\text { SetMarketProtocol }\end{array}$ \\
\hline
\end{tabular}

The domain knowledge is represented by three ontologies developed with the Protégé ontology editor: the MMB platform ontology, the viewer and program profiles ontology and the ad profile ontology. The MMB platform ontology is a Protégé frame ontology with the following main concepts: (i) AgentAction that contains all actions concerning the operations of Web services; (ii) AgentType that includes all types of agents used in the platform; and (iii) AgentData that holds the ads, intervals and viewers, including the corresponding profiles. The viewers and programs ontology is an OWL ontology inspired in the BBC program categories [6] and the ads ontology is an OWL ontology based on the Yellow Pages classified ads categories [8].

A viewer, program or ad profile is a vector of 15 features, where each feature corresponds to a category specified in the corresponding ontology. For example, the programs categories include Arts_and_Culture, History, Radio, News, Learning, Music, TV, Teens, Science_and_Nature, Entertainment, Sport, Health, Gardening, Weather and Food. The value of a profile feature varies from 0 (complete feature absence) to 9 (major feature presence). At runtime, the profile dimension cannot be altered, i.e., features cannot be added or suppressed, but features can be substituted. 


\subsection{Interface Layer}

The Interface Layer contains the layer manager agent (interface layer agent) and dedicated interface agents that support the interaction between external businesses and their platform representatives. The interface agents serve as intermediaries between enterprise layer agents and the external business interface applications.

\subsection{Enterprise Layer}

The Enterprise Layer holds the layer manager agent (enterprise layer agent) and the agents that model the businesses (distributor and producer enterprises) within the platform. The distributor agents are continuously trying to find and invite producer agents with ads matching the upcoming viewer intervals. Interval profiles are based on the context (the program being watched) and on the viewer profiles.

The matching between interval and ad profiles is performed by the producer agents in order to select the ads to negotiate in the market layer and, thus, decide whether to accept the distributor agent invitations. Since ads and intervals use distinct ontologies, it is necessary, before applying any algorithms, to map the ads and programs ontologies. The adopted mapping is straightforward two step mechanism: a search for ad features that are identical to program features followed by a final search for ad subfeatures that are identical to any unmapped program features. The matching mechanism is supported by a set of dedicated rules which use two distinct similarity algorithms to match ads with intervals profiles to rank the candidate ads. The first algorithm determines the similarity using the cosine similarity and the Euclidean distance [9]. The second algorithm computes the similarity based on the dominant characteristic of the interval. Depending on the resulting similarity ranking, the producer agents decide whether to accept the invitation to negotiate an ad and, consequently, launch a producer delegate in the market layer. The rules outcome is a similarity ranking between 1 (lowest) and 4 (highest).

\subsection{Market Layer}

The Market Layer contains the layer manager agent (market layer agent) and the enterprise delegate agents that represent the ads and intervals (distributor and producer delegates) under negotiation. Each delegate negotiates a single ad or interval on behalf of the corresponding producer or distributor agent according to the defined ad or interval negotiation behaviour: price range and adaptation tactic.

The market implements the Fixed ICNIP (FICNIP) negotiation protocol which is a variant of the Foundation for Intelligent Physical Agents (FIPA) Iterated Contract Net Interaction Protocol (ICNIP) [10]. While the FIPA-ICNIP stops as soon as there is an offer that matches the seller's target price, the FICNIP iterates for a fixed number of times regardless of the values of the buyers' offers received so far. 


\section{$3 \quad$ Tests and Results}

Three types of tests were conducted to assess the operation of the MMB platform: $(i)$ the ad selection mechanism; (ii) the negotiation of an interval involving a distributor and producers with different negotiation tactics and equal price variation ranges; and (iii) the negotiation of an interval involving a distributor and producers with different negotiation price variation ranges and equal adaptation tactics.

\subsection{Ad Selection}

This scenario is intended to illustrate the application of the similarity metrics implemented to select advertisements (the cosine similarity and dominant characteristic similarity) and involves a producer and a distributor enterprise. The producer enterprise submits two different ads. The distributor enterprise uploads to the platform an upcoming interval together with the corresponding viewer and context (current program) profiles. Table 2 displays the ad profiles.

Table 2. - Ad characteristics

\begin{tabular}{|l|l|c|c|c|}
\multicolumn{1}{l}{ Product } & \multicolumn{1}{c}{ Enterprise } & \multicolumn{1}{c}{ Timeslot $(\mathbf{s})$} & \multicolumn{1}{c}{ Profile } & \multicolumn{1}{c|}{ Ref. Price/s (€) } \\
\hline Ferrari & Prod001 & 30 & 897864156494888 & 10 \\
\hline Jaguar & Prod001 & 30 & 987489496848499 & 10 \\
\hline
\end{tabular}

Table 3 presents the viewer, context (program being watched) and resulting interval profiles held by the distributor agent.

Table 3. - Viewer, context and resulting interval profiles

\begin{tabular}{|c|c|c|c|c|c|}
\hline Viewer & Enterprise & Viewer Profile & Channel & Program & Program Profile \\
\hline 1 & Dist001 & 104351267334794 & Discovery & MythBusters & 826492673411245 \\
\hline Interval & Enterprise & Timeslot (s) & \multicolumn{2}{|c|}{ Ref. Price/s $(€)$} & Interval Profile \\
\hline 1 & Dist001 & 60 & & 25 & 415371465322464 \\
\hline
\end{tabular}

Table 4 holds the results of applying to both ads the matching rules based on the cosine similarity and on the interval dominant characteristic. In the first case, the producer agent chooses to negotiate the Ferrari ad and, in the latter case, chooses to negotiate the Jaguar ad. The ad ranking, which varies between a minimum of 1 and a maximum of 4 , is determined through defined matching rules. If a tie results from the application of a similarity metric, the producer chooses randomly one of the tied ads.

Table 4. - Matching rules results

\begin{tabular}{|c|c|c|c|c|c|c|}
\hline Product & Similarity & Distance & Ranking & Int. Dom. Char. & Ad Dom. Char. & Ranking \\
\hline Ferrari & 0.431 & 1.066 & 1 & 7 & 6 & 2 \\
\hline Jaguar & 0.379 & 1.114 & 1 & 7 & 8 & 3 \\
\hline
\end{tabular}

These results demonstrate the proper functioning of matching mechanism. 


\subsection{Price Adaptation Tactics}

This scenario involves one distributor and five producer enterprises. The producer enterprises submit the ads presented in Table 5. All ads have equal price variation ranges, but adopt different price adaptation tactics during the negotiation. The negotiation protocol is FICNIP.

Table 5. - Ads characteristics

\begin{tabular}{l|c|c|c|c|c|}
\cline { 2 - 6 } Product & Ferrari & Porsche & Toyota & LandRover & Mazda \\
\cline { 2 - 6 } $\begin{array}{l}\text { Enterprise } \\
\text { Price Tactic }\end{array}$ & Prod001 & Prod002 & Prod003 & Prod004 & Prod005 \\
\cline { 2 - 6 } Ref. Price/s (€) & Quadratic & Exponential & Linear & Random & Fixed \\
\cline { 2 - 6 } Max. Price/s (€) & 10 & 10 & 10 & 10 & 30 \\
\cline { 2 - 6 } Timeslot (s) & 50 & 50 & 50 & 50 & 50 \\
\cline { 2 - 6 } & 30 & 30 & 30 & 30 & 30 \\
\hline
\end{tabular}

The distributor enterprise uploads the interval features and corresponding viewer and context (current program) profiles. The viewer profile, viewer context profile and the resulting interval profile are presented in Table 6.

Table 6. - Viewer, context and resulting interval characteristics

\begin{tabular}{|c|c|c|c|c|c|}
\hline Viewer & Enterprise & Viewer Profile & Channel & Program & Program Profile \\
\hline 2 & Dist001 & 950567777851928 & Discovery & MythBusters & 826492673411245 \\
\hline r & $\mathbf{E}$ & im & $R$ & $(€)$ & le \\
\hline 2 & Dist001 & 150 & & 25 & 833474675631536 \\
\hline
\end{tabular}

The results of this interval negotiation are shown in Table 7 and correspond to the final interval content. They demonstrate the negotiation of a full interval involving producers with equal price ranges and diverse price adaptation tactics.

Table 7. - Interval 2 negotiation results

\begin{tabular}{l|c|c|c|c|c|}
\cline { 2 - 6 } Product & Ferrari & Porsche & Toyota & LandRover & Mazda \\
\cline { 2 - 6 } Negotiated Price/s (€) & 50.0 & 50.0 & 45.0 & 49.3 & 30.0 \\
\cline { 2 - 6 }
\end{tabular}

\subsection{Price Ranges}

This scenario illustrates the FICNIP negotiation behaviour with ads with the same adaptation tactics and different price ranges. It involves one distributor and two producer enterprises. The producer ad parameters are presented in Table 8.

Table 8. - Ads characteristics

\begin{tabular}{l|c|c|}
\cline { 2 - 3 } Product & Jaguar & Porsche \\
\cline { 2 - 3 } Enterprise & Prod001 & Prod002 \\
\cline { 2 - 3 } Raxice/s (€) & 15 & 10 \\
\cline { 2 - 3 } Mrice/s (€) & 90 & 50 \\
\cline { 2 - 3 } Price Tactic & Exponential & Exponential \\
\cline { 2 - 3 } Timeslot (s) & 60 & 60 \\
\hline
\end{tabular}


The distributor enterprise uploads the viewer and viewer context (current program) profiles and upcoming viewer interval features. Table 9 presents the uploaded data and the resulting interval profile.

Table 9. - Viewer, context and resulting interval profiles

\begin{tabular}{|c|c|c|c|c|c|}
\hline Viewer & Enterprise & Viewer Profile & Channel & Program & Program Profile \\
\hline 2 & Dist001 & 950567777851928 & Discovery & MythBusters & 826492673411245 \\
\hline Interv & & eesl & & E) & \\
\hline 3 & Dist001 & 60 & & 25 & 833474675631536 \\
\hline
\end{tabular}

The negotiation results are shown in Table 10. These results demonstrate a negotiation involving two producers with the same price adaptation tactics and different price ranges. The Porsche ad was not added to the interval because it was full after the successful negotiation of the Jaguar ad.

Table 10. - Interval 3 negotiation results

\begin{tabular}{l|c|} 
Product & Jaguar \\
\cline { 2 - 2 } Negotiated Price/s (€) & 90.0 \\
\hline
\end{tabular}

\section{Conclusions}

This paper presents the application domain, the developed platform functionalities as well as three different test scenarios that illustrate the platform operation.

In terms of achievements, the developed MMB platform prototype is able to trade timeslots between content producers and distributors based on the viewer, program, interval and ad profiles. The architecture is organized into three layers: $(i)$ interface layer that is responsible for interacting with the external businesses; (ii) enterprise layer where distributor invite producer agents to negotiate and producer agents select ads for negotiation based on the profile matching mechanism; and (iii) market layer, where delegate agents of the intermediate layer agents meet to negotiate interval timeslots according to the ascribed ad negotiation behaviour (price range and adaptation tactic). The communication between agents of different layers and between the platform and the external entities is performed through Web services. The market agents communicate by exchanging FIPA-ACL messages and implement the Fixed ICNIP (FICNIP) negotiation protocol. All Web services exposed by the agents are published in a UDDI registry for discovery and consumption.

Concerning future developments, the mapping between the programs and ads profile ontologies can be refined. Currently it implements a direct mapping between the corresponding features of programs and ads and between the remaining unmatched program features and the corresponding ad sub-features. Different weights should be attributed to features and sub-features and, in the case of several matching subfeatures, the mean value should be attributed instead of the highest value currently used. The matching mechanism between ad and interval profiles can be enhanced. The producer agents select the ads based on the similarity between ad and interval profiles. The similarity based on the interval dominant characteristic can use, instead 
of a single dominant characteristic, the top five interval characteristics (one third of the profile features). Furthermore, unexpected ads should be regularly chosen, provoking a sense of novelty and unpredictability on the viewer, i.e., introducing serendipity. Finally, the platform evaluation needs to be carried out with real users and data.

\section{Acknowledgment}

This work is financed by the ERDF - European Regional Development Fund through the COMPETE Programme (operational programme for competitiveness) and by National Funds through the FCT - Fundação para a Ciência e a Tecnologia (Portuguese Foundation for Science and Technology) within project «FCOMP - 01-0124FEDER-022701».

\section{References}

1. M. López-Nores et al., "MiSPOT: dynamic product placement for digital TV through MPEG-4 processing and semantic reasoning," Knowledge and Information Systems, vol. 22, no. 1, pp. 101-128, 2010.

2. NoTube, "NoTube," 2012. [Online]. Available: http://notube.tv/. [Accessed in February 2013].

3. LinkedTV, "LinkedTV," 2012. [Online]. Available: http://www.linkedtv.eu/. [Accessed in February 2013].

4. HBB-NEXT, "HBB-NEXT Next Generation Media," 2012. [Online]. Available: http://www.hbb-next.eu/index.php. [Accessed in February 2013].

5. L. V. de Sousa, B. Malheiro and J. Foss, "Negotiation platform for personalised advertising," in Proceedings of the Fifth International European Conference on the Use of Modern Information and Communication Technologies (ECUMICT 2012), L. de Strycker, Ed., 2012, pp. 361-373.

6. J. D. Foss, B. Malheiro and J. C. Burguillo, "Personalised placement in networked video," in Proceedings of the $21^{\text {st }}$ international conference companion on World Wide Web, ser. WWW'12 Companion. New York, NY, USA: ACM, 2012, pp. 959-968.

7. British Broadcasting Corporation. Program categories. [Online]. Available: http://www.bbc.co.uk/a-z/. [Accessed in September 2012].

8. Yellow Pages. Classified ads categories. [Online]. Available: http://www.yellowpages.com/. [Accessed in September 2012].

9. A. Madylova and S. Oguducu, "A taxonomy based semantic similarity of documents using the cosine measure," in Proceedings of the $24^{\text {th }}$ International Symposium on Computer and Information Sciences (ISCIS 2009), 2009, pp. 129-134.

10. Foundation for Intelligent Physical Agents, "FIPA iterated contract net interaction protocol specification," FIPA TC Communication, Standard 30, 2002. [Online]. Available: http://www.fipa.org/specs/fipa00030/SC00030H.pdf. [Accessed in September 2012]. 